\title{
EDM OF ALUMINUM ALLOY 6061 USING GRAPHITE ELECTRODE USING PARAFFIN OIL AND DISTILLED WATER AS DIELECTRIC MEDIUM
}

\author{
Muhammad Imran', Sayyid Masoodur Rahman shah'1, Shahid Mehmood', Rubab Arshad' \\ 1 University of Engineering and Technology, 47050 Taxila, Pakistan, e-mail: shahid.mehmood@uettaxila.edu.pk
}

Received: 2017.05.15 Accepted: 2017.08.01 Published: 2017.09.03

\begin{abstract}
EDM machining of $\mathrm{Al} 6061$ was performed under varying conditions of pulse current and pulse duration. Graphite was used as an electrode material with distilled water and paraffin oil as two different dielectric mediums. The aim is to characterize the surface integrity produced as a result of EDM machining under both varying electrical and non-electrical parameters as it is important in determining the service life of EDM machined components. The vertical, horizontal and corner surfaces are studied independently for each single set of conditions. The average white layer thickness (AWLT) and surface roughness was found to be dependent on pulse current values and pulse duration. The dielectric medium also has an influence on the thickness and nature of white layer. It was found that the thickness and nature of the white layer formed from distilled water is different from that of paraffin oil. New methods of AWLT and surface roughness measurement through an optical microscope are described. The surface roughness method developed was calibrated against an identical surface roughness tester. Finally, material removal rate, tool wear rate, the presence of micro cracks, voids and globules were compared for different conditions and conclusions were drawn according to the actual physical conditions during machining.
\end{abstract}

Keywords: EDM, white layer, surface roughness, material removal rate, micro cracks, surface integrity.

\section{INTRODUCTION}

Electric Discharge Machine (EDM) is nonconventional machining process used to machine conductive material using thermal properties of electric discharge current. EDM uses thermal energy of electric current to remove material through an erosion process of electrically conductive material [14]. Erosive properties of electric discharge was invented by English chemist Joseph Priestly in 1770 and in 1943, Russian scientists R.B. Lazarenko and N.I. Lazarenko used the damaging properties of electric discharge for useful purposes [4]. In the EDM process, the thermal energy of electric discharge removes the material. A high temperature electrical spark is produced and melts the material or work-piece. The molten material is removed by dielectric flushing. A part of molten material is removed after each spark, and the rest of the material is re-solidified on the EDM machined surface or work-piece to form a layer. This layer after machining is called white layer (WL) or recast layer (RL) $[1,12]$. Four layers are observed after EDM machining. The first one is a debris layer, the second is the white layer, the third one is an annealed layer or heat affected zone and on the final layer parent material can be observed $[9,18]$. The formation of white layer and its thickness is dependent on both the electrical and non-electrical EDM parameter [13]. Pulse duration and pulse current are the key aspects of spark energy that effects MRR and surface roughness $[15,19]$. 
The composition and properties of white layer differs from the base material because this layer is formed in the presence of dielectric medium at high temperatures. Some of the foreign atoms from electrode and dielectric are induced into the recast layer which changes the composition of white layer from the parent material $[10,12]$.

Surface cracks are surface defects which are generated from white layer surface and penetrate into heat affect zone of the EDM machined surface [20]. The density of surface cracks and AWLT has a direct relation with work-piece thickness and inverse relation with thermal conductivity because greater thermal conductivity increases the heat distribution equally [17]. It is found that these cracks directly related to the pulse duration and inversely related to the pulse current [5].

Dielectric medium act as an insulator material before ionization between the work-piece and electrode after sparking, which provide cooling, and removes melted material. Hydrocarbon oil, usually paraffin oil and distilled water dielectric medium are used. Paraffin oil has greater arcing stability while distilled water has better surface finish [2]. Debris and bubbles are removed by dielectric flushing and its formation has direct relation with pulse duration and pulse current [21]. It was found that every working fluid has its own individual features and outcomes [8]. Using oil based dielectrics mediums increased the amount of carbon in the recast layer steel with better MRR. While the water-based dielectric fluids reduces the amount of carbon with lesser surface cracks, density and thicker heat affected zone as compared to oil base dielectrics. Tool wear ratio is almost zero and MRR is high when EDM is done with gas as dielectric and with the increase of ox- ygen concentration in air, the MRR increased [6, 7]. The dielectric fluids used for EDM and their effects on generated surface were widely evaluated by S. Chakraborty et al. [2]. Distilled water as compared to paraffin oil shows greater MRR and lower EWR. Distilled water produces better surface finish than paraffin oil. Better results can be obtained at high pulse duration and pulse current and a mixture of carbon based components with distilled water. The strength of dielectric and open circuit is decreased, which increases the gap between the tool and work-piece by using metallic powder mix dielectric. It enhances the stability of the process, MRR and surface quality [3].

Initially EDM were used in die production industry, where hard materials such as steel tools, tungsten and tungsten carbides are difficult to machine by conventional methods. In recent times lighter metals other than ferrous alloys are easily machined by EDM such as titanium alloys and nickel based alloys [16]. EDM is also typically used to machine soft material like aluminum alloys. The main reasons for using EDM for the machining of soft alloys are 1) hard to reach points: - long EDM electrode is sometimes the only possible method, 2) complex geometries: long unsighted rectangular holes or inner splines are sometimes only possible through EDM and 3) very little work: micro EDM [11].

Much work has been carried out on tool steel and other grades of steel. High strength aluminum alloys have many applications in the aerospace and automobile industry because of their high strength to weight ratio. Therefore, the current study is performed to explore the altered characteristics of the aluminum alloy after EDM, under different conditions of EDM.

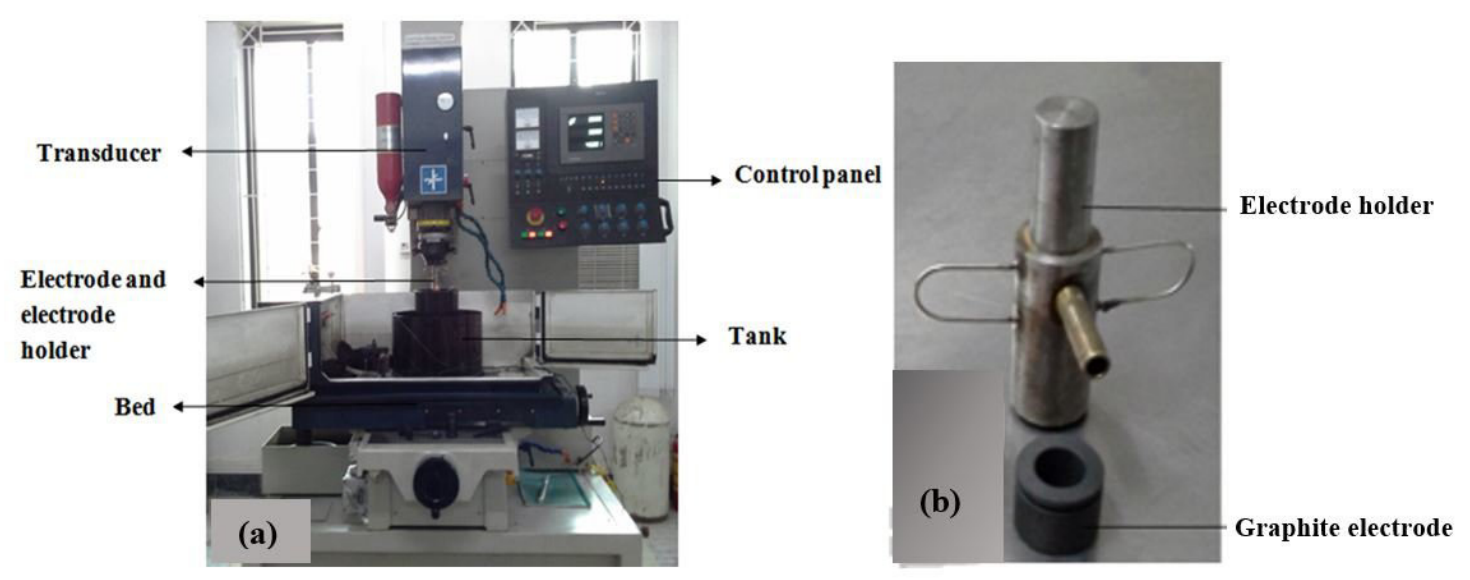

Fig. 1. EDM machining with distilled water (left) and graphite electrode with its fixture (right) 
Table 1. Experimental conditions in EDM

\begin{tabular}{|l|c|}
\hline Pulse current $(\mathrm{A})$ & 6,9, and 12 \\
\hline Gap $($ mil) & 10 \\
\hline Pulse duration $(\mu \mathrm{s})$ & $60,90,120,150$, and 200 \\
\hline Pulse off time $(\mu \mathrm{s})$ & 3 \\
\hline
\end{tabular}

\section{EXPERIMENTAL SETUP AND METHODOLOGY}

The Experiments are performed using NEUAR sinking EDM with thick hollow electrodes. The hollow shape of the electrode produces the horizontal (HS), vertical (VS) and corner between horizontal and vertical surface (CS). EDM machined surface with distilled water is performed in a separate tank with a water pumping system so that paraffin oil would not contaminate the distilled water as shown in Figure 1.

Same machining conditions are set during experimentation for graphite electrode with paraffin oil and distilled water dielectrics as presented in Table 1.

Thirty samples are machined, and the design of experimentation is given in Table 2. Using metallography, samples are prepared for optical microscopy to observe the cross section view of the machined surface. Recast surface is observed under optical microscope OLYMPUS BX51 at $50 \mathrm{X}$ and $20-\mu \mathrm{m}$ resolution and all three surfaces are considered. AWLT is calculated by using image processing technique module Image $J$ as shown in Figure 2. AWLT is calculated by dividing area of white layer by its length. For each sample, 6-10 values of AWLT are taken at different machined surfaces including horizontal, vertical and curved surface between these two. Finally, the mean value of both horizontal and vertical surfaces is taken.

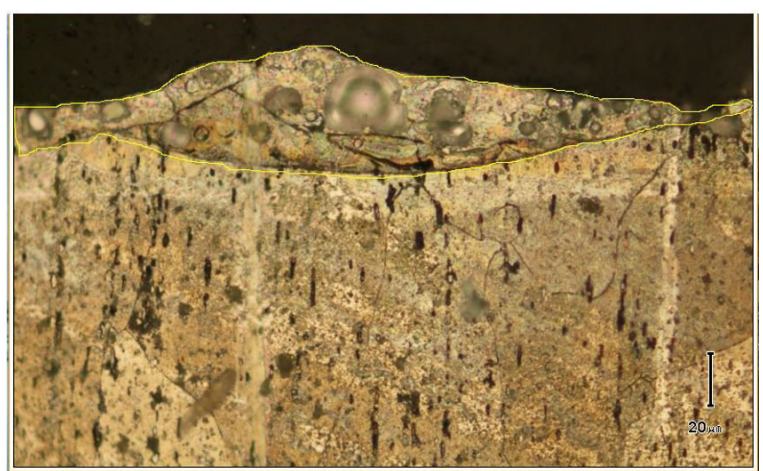

Fig. 2. Selected area for AWLT
Table 2. Design of Experiment for EDM

\begin{tabular}{|c|c|c|}
\hline \multirow{2}{*}{$\begin{array}{l}\text { Pulse } \\
\text { Current } \\
\text { (A) }\end{array}$} & $\begin{array}{l}\text { Graphite electrode } \\
\text { with paraffin oil }\end{array}$ & $\begin{array}{l}\text { Graphite electrode } \\
\text { with distilled water }\end{array}$ \\
\hline & Pon ( $\mu s)$ & Pon $(\mu \mathrm{s})$ \\
\hline \multirow{5}{*}{$\begin{array}{l}\text { Level } \\
\text { (1) } \\
=6 \mathrm{~A}\end{array}$} & 60 & 60 \\
\hline & 90 & 90 \\
\hline & 120 & 120 \\
\hline & 150 & 150 \\
\hline & 200 & 200 \\
\hline \multirow{5}{*}{$\begin{array}{l}\text { Level } \\
\quad(2) \\
=9 \mathrm{~A}\end{array}$} & 60 & 60 \\
\hline & 90 & 90 \\
\hline & 120 & 120 \\
\hline & 150 & 150 \\
\hline & 200 & 200 \\
\hline \multirow{5}{*}{$\begin{array}{l}\text { Level } \\
\begin{array}{c}(3) \\
=12 \mathrm{~A}\end{array}\end{array}$} & 60 & 60 \\
\hline & 90 & 90 \\
\hline & 120 & 120 \\
\hline & 150 & 150 \\
\hline & 200 & 200 \\
\hline
\end{tabular}

Surface roughness is measured by selecting surface profile using Engauge Digitizer 2.12 module. In Engauge Digitizer we define scale and coordinate system and mark the set of data points on the curve profile as shown in Figure 4.

These set of data points are imported into Microsoft Excel and the surface roughness ( $\mathrm{Ra}$ ) is measured by with the following equation:

$$
\mathrm{Ra}=\frac{1}{\mathrm{n}} \sum_{\mathrm{i}=1}^{\mathrm{n}}|\mathrm{yi}-\mathrm{m}|
$$

where: $y=$ height of crest/trough from reference line, $\mathrm{m}=$ height of mean line from reference line, $\mathrm{n}=$ total number of data points.

For each sample the surface roughness is measured 10 times, 5 times for horizontal surface and 5 times for vertical surface at various points

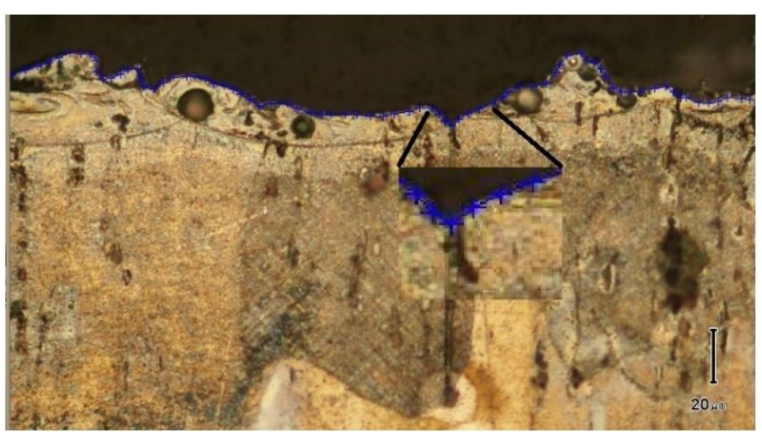

Fig. 3. Surface roughness measurement using Engauge Digitizer 


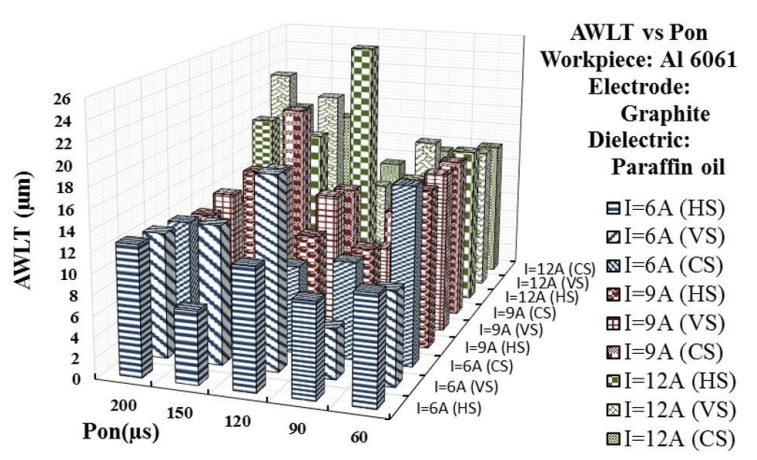

Fig. 4. AWLT variation with respect to pulse current and pulse duration

along the machined surface, and finally mean value of $\mathrm{Ra}$ is taken for each horizontal and vertical surfaces. These values of surface roughness are compared to the conventional portable surface roughness tester (Mitutoyo SJ-410). The coefficient correlation between surface roughnesses is measured through a digital image process, and conventional roughness tester is 0.86 and percentage error is about $10 \%$, which can be vibrational effect of environment.

\section{RESULTS AND DISCUSSION}

The results of the machined material such as white layer thickness, morphology, surface roughness and machining features like MRR (Material Removal Rate) and TWR (Tool Wear Rate) are presented in this section.

\section{White Layer Thickness for Paraffin Oil as Dielectric Medium}

The behavior of AWLT by changing pulse current and pulse duration is represented in Figure.4. It shows that all three horizontal, vertical and corner surfaces presenting AWLT value in up and down manners with the increase of pulse duration at various pulse current. At $120 \mu$ s pulse duration and $12 \mathrm{~A}$ pulse current, the horizontal and vertical surfaces are much difference in AWLT values which may due to improper flushing and defamation of white layer respectively. Similarly, AWLT values decreases as Pon increases from $60 \mu$ s to $90 \mu$ s then slightly increases with abrupt changes in horizontal, vertical and corner surfaces of white layer values as Pon varies from $90 \mu$ s to $200 \mu \mathrm{s}$.

Such irregular behavior indicates that at higher pulse energy total spark energy becomes so high, which increases possibility of over lapping the craters forming multiple white layers. The abrupt changing in white layer is due to improper flushing or deformation of white layer (like normal distribution) as shown in Figure 5. Figure 5(a) shows the breakdown of white layer with the formation of large cavities, due to trapped air, with micro cracks in white layer and penetrating into the heat affected zone. Figure 5(b) shows the formation of large size globules with cavities and micro cracks with overlapping of white layer as well as abrupt brakes in the white layer at $12 \mathrm{~A}$ current and $200 \mu$ s Pon. Figure 5 (c) shows moun-

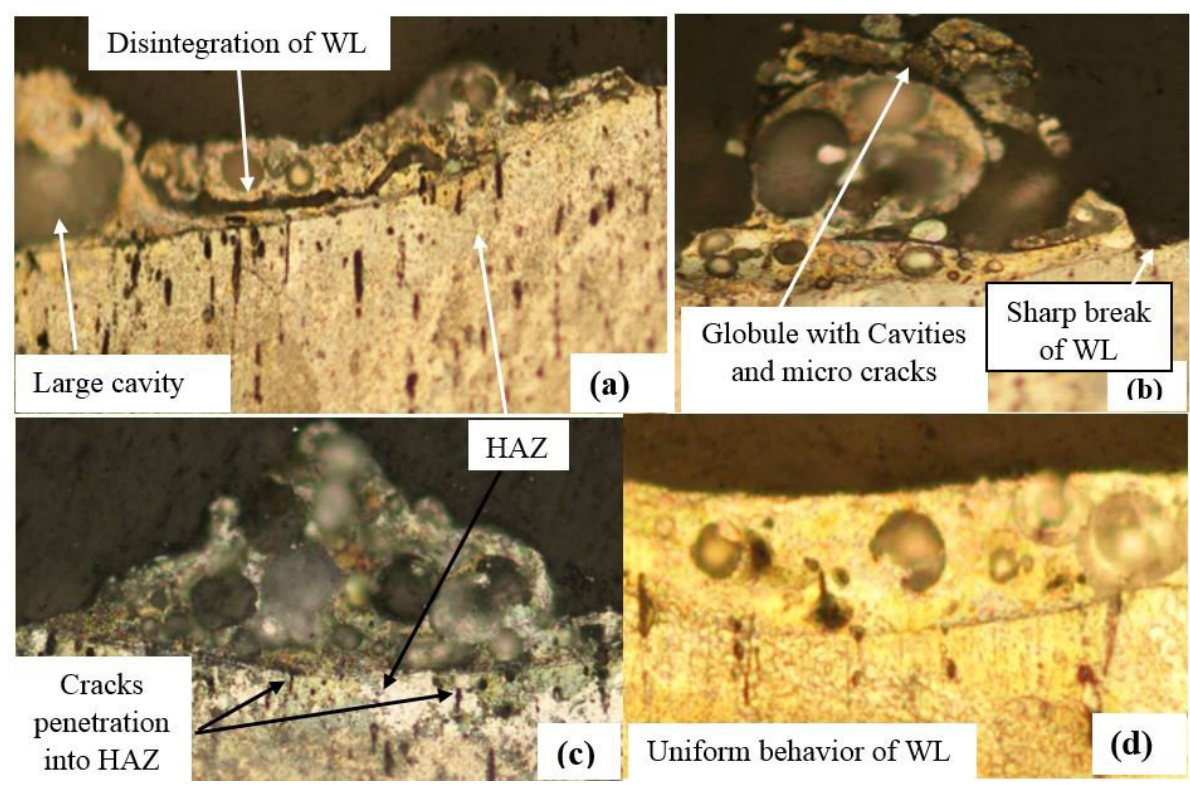

Fig. 5. cross sectional view of white layer; dielectric medium: parafine oil (a) and (b) are I=12A and 200 $\mu$ s Pon, (c) $\mathrm{I}=12 \mathrm{~A}$ and $150 \mu \mathrm{s}$ Pon and (d) $\mathrm{I}=9 \mathrm{~A}$ and $60 \mu \mathrm{s}(50 \mathrm{X}+\mathrm{crop}+$ resize $)$ 


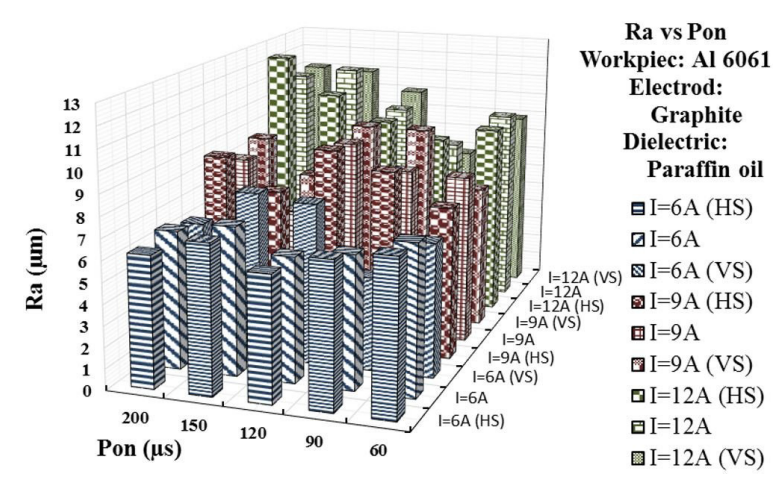

Fig. 6. Surface roughness behavior at different condition of pulse current and pulse duration

tainous like shapes with micro cracks penetrating into the heat affected zone, this can also indicate the improper flushing and deformation of white layer at $150 \mu$ s Pon and 12 A current. Figure 5 (d) shows uniform or smooth behavior of white layer because of a lower pulse current of $9 \mathrm{~A}$ at a lower pulse duration of $60 \mu \mathrm{s}$. Therefore, the zigzag manner of AWLT values with increasing Pon and pulse current is due to the breakage of white layer and improper flushing. Similarly, surface roughness values are slightly decrease then started to increase slightly with the increase of pulse duration as in Figure 6. It means the first breakage of white layer started when both improper flushing and disintigeration occured simultaneously, due to ovelappining of wihite layer as the pusle duration increases.

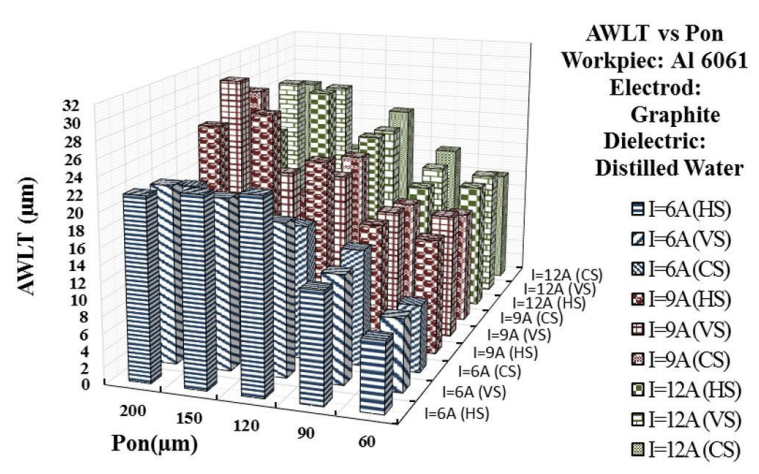

Fig. 7. AWLT variation with respect to pulse current and pulse duration

\section{Surface Roughness for Paraffin Oil as Dielectric Liquid}

The horizontal surface roughness values using Engauge digitizer ${ }^{\circledR}$ are compared with the conventional surface roughness tester as shown in Figure 6. The coefficient of correlation between surface roughness measured from digital image process, conventional roughness tester is 0.86 , and percentage error is about $10 \%$, which can be vibrational effect of environment.

\section{White Layer Thickness for Distilled water as dielectric medium}

The behavior of white layer formation is different from the white layer formation in paraffin oil. An increasing trend is observed with the
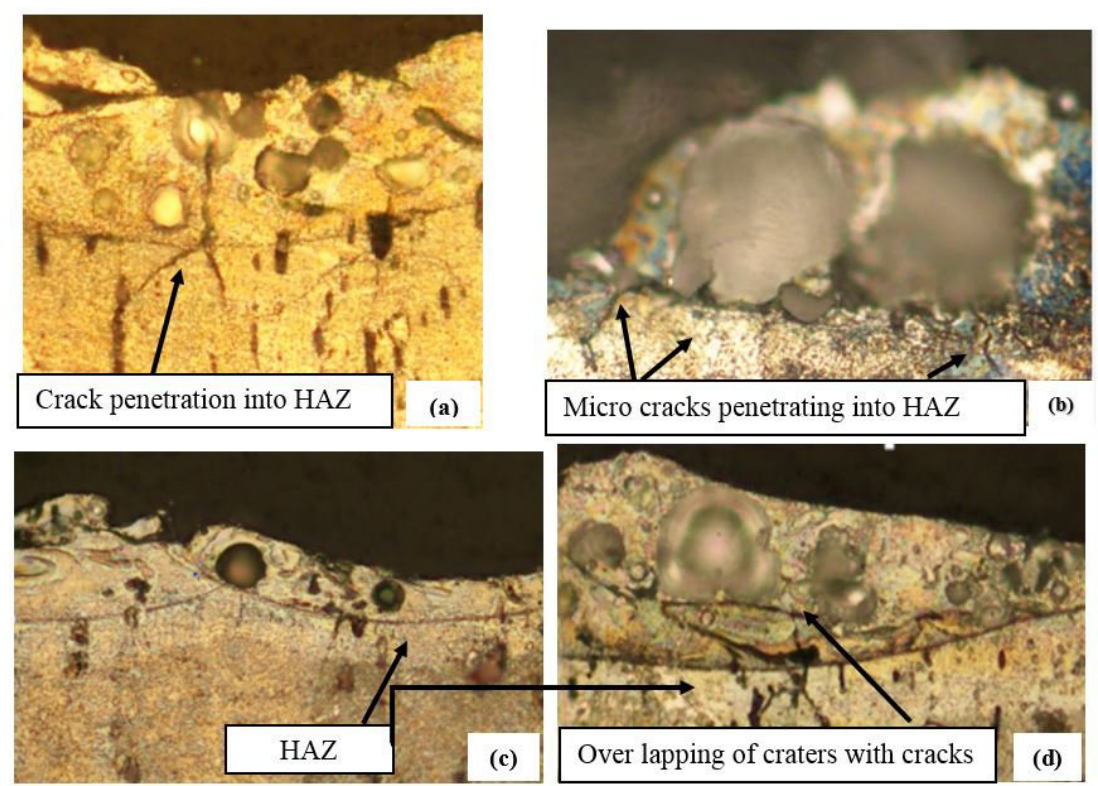

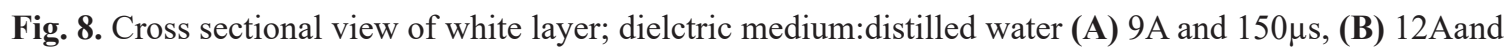
$200 \mu \mathrm{s}$, (C) $12 \mathrm{~A}$ and $60 \mu \mathrm{s}$, and (D) $12 \mathrm{~A}$ and $150 \mu \mathrm{s}$ Pon, $(50 \mathrm{X}+\mathrm{crop})$ 
increase of pulse current and pulse duration as shown in Figure 7.

It is observed that AWLT values for 9A and $12 \mathrm{~A}$ current are almost equal and increasing trend with the increase of pulse duration. The AWLT at $9 \mathrm{~A}$ current is greater than $12 \mathrm{~A}$ current as the pulse duration increases from $120 \mu \mathrm{s}$ to $200 \mu \mathrm{s}$. When AWLT at 6A current is increased from $60 \mu$ s to $90 \mu \mathrm{s}$ it becomes almost constant as of pulse duration is further increased. It shows even though breakage of the white layer takes place at high pulse current and pulse duration, this breakdown is lower than when paraffin oil is used as a dielectric, which can be seen through cross sectional view of white layer as shown in Figure 8. It can be seen through cross sectional view that the intensity of crack formation and penetration into the heat-affected zone is increased with the increase of pulse current and pulse duration. The magnitude of AWLT is observed greater using distilled water with graphite then paraffin oil.

The luster of heat-affected zone is clearer as compared to paraffin oil. A cross section view of Figure. 8 shows the densely packed WL with smooth surface finish of white layer, which can be observed through surface roughness behavior as represented in Figure.9.

\section{Surface Roughness for Distilled Water as Dielectric Medium}

As shown in Figure 9, a slight increase in surface roughness values is observed with the increase of pulse duration. However, the vertical surface at 12A current shows up and down behavior with the increase of pulse duration. It is also observed that the magnitude of surface roughness is much lower than surface roughness values obtain using paraffin oil as a dielectric.

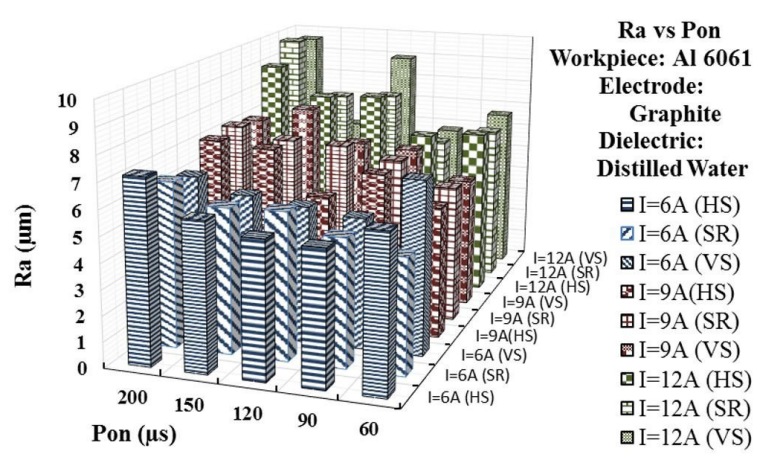

Fig. 9. Surface roughness behavior at different condition of pulse current and pulse duration

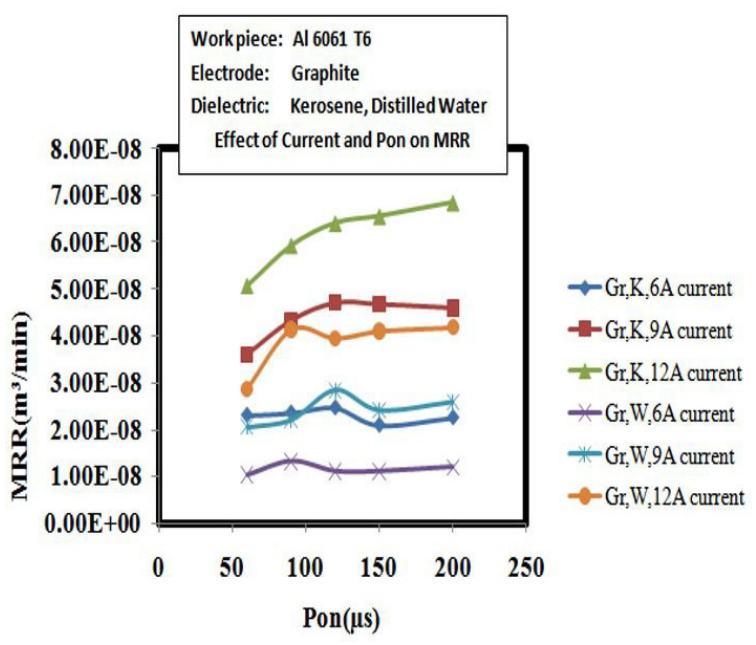

Fig. 10. MMR of Al-6061 for kerosene oil and distilled water as dielectric medium

\section{Material Removal Rate}

Weight difference in the before and after machining of specimens and electrode determines Material Removal Rate (MRR) and Electrode Wear Rate (EWR) respectively. Material Removal Rate is obtained during the machining of Al alloy with graphite electrodes using paraffin oil as dielectric as it has greater value than distilled water as shown in Figure 10. It is clearly seen that Material Removal Rate increases with the discharge current for both types of dielectric liquids. However, the Material Removal Rate is higher when Paraffin oil was used as a dielectric liquid as compared to distilled water. The variation in Pulse-ON time is effective on the Material Removal Rate for 9 and 12 ampere.

\section{Electrode Wear Rate}

Due to the porous nature of graphite, the dielectric liquid is absorbed into the electrode material and increases its weight after EDM that causes difficulty in measuring Tool Wear Rate (TWR). Therefore, the graphite electrode was put into an oven at $120{ }^{\circ} \mathrm{C}$ for 1 hour to evaporate any Paraffin oil absorbed in it. The results show that the EWR of graphite electrode with distilled water increases up to a pulse duration of $120 \mu \mathrm{s}$ and then starts decreasing. EWR with paraffin oil shows decreasing trend initially with the increase of pulse duration then slightly increased as represented in Figure 11. Apparently, it is clear that the electrode wear rate is more for distilled water as compared to Paraffin oil. 


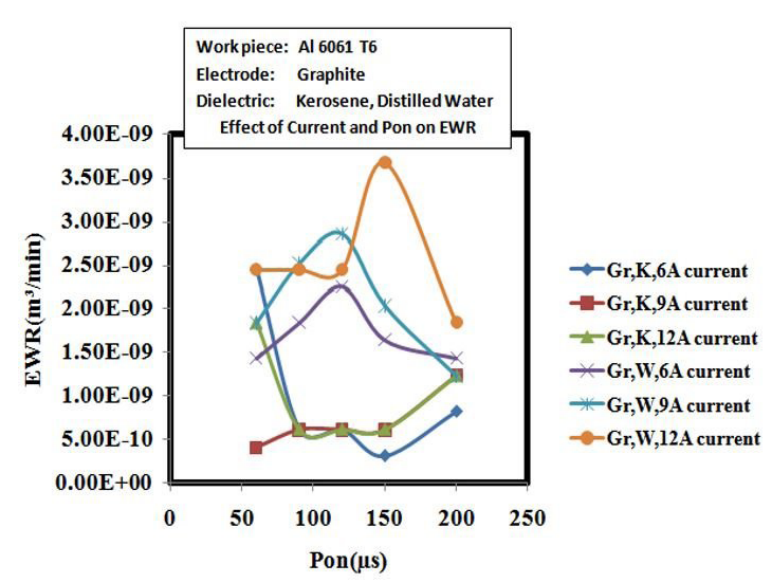

Fig. 11. EWR of graphite electrode in kerosene oil and distilled water as dielectric

\section{CONCLUSION}

The effect of graphite electrodes is determined by two different dielectric liquids for the electro-discharge machining of aerospace grade aluminum alloy 6061. This effect was observed for the important electric parameters such as discharge current and pulse-ON time. The influence of EDM is determined by the thickness of the white layer, surface roughness and surface morphology. The findings of this experimental work are:

- The thickness of the white layer is found more with the increasing value of Pulse On-Time and discharge current. The effect of dielectric liquid on the thickness of the white layer is also observed. A thicker white layer is obtained using distilled water as a dielectric medium as compared to paraffin oil by applying the same machining conditions during EDM. Paraffin oil also provides a rougher surface as compared to distilled water that is because of the reactive nature of the Paraffin oil causing severe surface pits and larger globules. The white layer generated by the use of paraffin oil is of porous nature and of that produced using distilled water with the same graphite electrode and other machining condition is dense packed.

- The intensity of the surface cracks and thickness of heat-affected zone is found more from the use of distilled water as compared to paraffin oil and is increasing with pulse duration and discharge current. This effect was attributed to the higher thermal conductivity of distilled water causing rapid cooling of the mol- ten material resulting severe surface cracks.

- The paraffin oil gives higher MRR and lower EWR as compared to distilled water that was also found dependent on discharge current and pulse-On time.

\section{Acknowledgements}

The authors would like to acknowledge the faculty and the very helpful staff of the Fracture Mechanics Laboratory in Mechanical Engineering Department of University of Engineering and Technology Taxila, Pakistan.

\section{REFERENCES}

1. Arooj, S., M. Shah, S. Sadiq, S.H.I. Jaffery, and S. Khushnood, Effect of Current in the EDM Machining of Aluminum 6061 T6 and its Effect on the Surface Morphology. Arabian Journal for Science and Engineering. 39(5): 2014. 4187-4199.

2. Chakraborty, S., V. Dey, and S.K. Ghosh, A Review on the Use of Dielectric Fluids and their Effects in Electrical Discharge Machining Characteristics. Precision Engineering. 40: 2015. 1-6.

3. Garg, R.K., K.K. Singh, A. Sachdeva, V.S. Sharma, K. Ojha, and S. Singh, Review of research work in sinking EDM and WEDM on metal matrix composite materials. The International Journal of Advanced Manufacturing Technology. 50(5-8): 2010. 611-624.

4. Ho, K.H. and S.T. Newman, State of the Art Electrical Discharge Machining (EDM). International Journal of Machine Tools and Manufacture. 43(13): 2003. 1287-1300.

5. Jha, A.K., K. Sreekumar, and P.P. Sinha, Role of electro-discharge machining on the fatigue performance of 15-5PH stainless steel component. Engineering Failure Analysis. 17(5): 2010. 1195-1204.

6. Kunieda, M., S. Furuoya, and N. Taniguchi, Improvement of EDM Efficiency by Supplying Oxygen Gas into Gap. CIRP Annals - Manufacturing Technology. 40(1): 1991. 215-218.

7. Kunieda, M., M. Yoshida, and N. Taniguchi, Electrical Discharge Machining in Gas. CIRP Annals Manufacturing Technology. 46(1): 1997. 143-146.

8. Leão, F.N. and I.R. Pashby, A review on the use of environmentally-friendly dielectric fluids in electrical discharge machining. Journal of Materials Processing Technology. 149(1-3): 2004. 341-346.

9. Lee, H.-T., F.-C. Hsu, and T.-Y. Tai, Study of surface integrity using the small area EDM process with a copper-tungsten electrode. Materials Science and Engineering: A. 364(1-2): 2004. 346-356. 
10. Lee, S.H. and X. Li, Study of the surface integrity of the machined workpiece in the EDM of tungsten carbide. Journal of Materials Processing Technology. 139(1-3): 2003. 315-321.

11. M. Pawade, M. and S. S. Banwait, A Brief Review of Die Sinking Electrical Discharging Machining Process towards Automation. American Journal of Mechanical Engineering. 1(2): 2013. 43-49.

12. Mehmood, S., R.A. Pasha, and A. Sultan, Effect of Electric Discharge Machining on Material Removal Rate and white layer composition. Mehran University Research Journal of Engineering \& Technology. 36(1): 2017. 45-54.

13. Mehmood, S., M. Shah, R.A. Pasha, S. Khushnood, and A. Sultan, Influence of electric discharge machining on fatigue limit of high strength aluminum alloy under finish machining. Journal of the Chinese Institute of Engineers. 40(2): 2017. 118-125.

14. Mehmood, S., A. Sultan, N.A. Anjum, W. Anwar, and Z. Butt, Determination of residual stress distribution in high strength aluminum alloy after edm. Advances in Science and Technology Research Journal. 11(1): 2017. 29-35.

15. Muthuramalingam, T. and B. Mohan, A review on influence of electrical process parameters in EDM process. Archives of Civil and Mechanical Engineering. 15(1): 2015. 87-94.
16. Pandey, A. and S. Singh, Current research trends in variants of Electrical Discharge Machining: A review. International Journal of Engineering Science and Technology. 2(6): 2010. 2172-2191.

17. Ramasawmy, H., L. Blunt, and K.P. Rajurkar, Investigation of the relationship between the white layer thickness and 3D surface texture parameters in the die sinking EDM process. Precision Engineering. 29(4): 2005. 479-490.

18. Shabgard, M., S.N.B. Oliaei, M. Seyedzavvar, and A. Najadebrahimi, Experimental investigation and 3D finite element prediction of the white layer thickness, heat affected zone, and surface roughness in EDM process. Journal of Mechanical Science and Technology. 25(12): 2012. 3173-3183.

19. Shabgard, M., M. Seyedzavvar, and S.N.B. Oliaei, Influence of Input Parameters on the Characteristics of the EDM Process. Strojniški Vestnik - Journal of Mechanical Engineering. 57(9): 2011. 689-696.

20. Tai, T. and S. Lu, Improving the Fatigue Life of Electro-Discharge-Machined SDK11 Tool Steel via the Suppression of Surface Cracks. International Journal of Fatigue. 31(3): 2009. 433-438.

21. Wang, J. and F. Han, Simulation model of debris and bubble movement in consecutive-pulse discharge of electrical discharge machining. International Journal of Machine Tools and Manufacture. 77: 2014. 56-65. 